Journal of Astronomical Instrumentation

(C) World Scientific Publishing Company

\title{
A Data-Taking System for Planetary Radar Applications
}

\author{
Jean-Luc Margot ${ }^{1,2}$ \\ ${ }^{1}$ Department of Earth, Planetary, 85 Space Sciences, UCLA, Los Angeles, CA 90095, USA, jlm@epss.ucla.edu \\ ${ }^{2}$ Department of Physics 6 Astronomy, UCLA, Los Angeles, CA 90095, USA, jlm@astro.ucla.edu
}

Received October 23, 2020; Revised November 17, 2020; Accepted November 24, 2020;

\begin{abstract}
Most planetary radar applications require recording of complex voltages at sampling rates of up to $20 \mathrm{MHz}$. I describe the design and implementation of a sampling system that has been installed at the Arecibo Observatory, Goldstone Solar System Radar, and Green Bank Telescope. After many years of operation, these data-taking systems have enabled the acquisition of hundreds of data sets, many of which still await publication.

Keywords: Radar; Planets; Asteroids; Data acquisition.
\end{abstract}

\section{Motivation}

Planetary radar astronomy is a discipline that enabled major advances in our understanding of the scale of the Solar System, spin states and surface properties of planets and satellites, and the physical and dynamical properties of asteroids (e.g., Ostro et al., 2000; Giorgini et al., 2002; Margot et al., 2002; Chesley et al., 2003; Campbell et al., 2006; Ostro et al., 2006; Margot et al., 2007; Taylor et al., 2007). For reviews of the field, see Ostro (1993); Campbell et al. (2002); Ostro et al. (2002); Benner et al. (2015). For an historical account of the development of the field up to 1996, see Butrica (1996).

The field is distinctive in part because essentially all results come from only two transmitting facilities, the Arecibo Planetary Radar and the Goldstone Solar System Radar. The facilities are complementary in that the former is $\sim 15$ times more sensitive and the latter has access to a fraction of the sky that is $\sim 2.5$ larger (Naidu et al., 2016). The facilities also probe slightly different depths below the surface because Arecibo transmits primarily at $\mathrm{S}$ band $(2380 \mathrm{MHz})$ whereas Goldstone transmits primarily at $\mathrm{X}$ band (8560 MHz). Bistatic observations with reception at the $100 \mathrm{~m}$ Green Bank Telescope (GBT) or other antennas also occur for a small fraction of the observations.

Most planetary radar observations yield either 1D power spectra or 2D range-Doppler images (Evans \& Hagfors, 1968), although 3D topographic maps can be obtained in some circumstances (Margot et al., 1999). The sampling rate requirements for power spectra are relatively modest $(<60 \mathrm{kHz})$ because the Doppler broadening of Solar System bodies at $\mathrm{S}$ band and $\mathrm{X}$ band do not exceed $30 \mathrm{kHz}$, with the exception of Saturn's rings (Nicholson et al., 2005). For 2D imaging, the transmitted waveforms are most commonly encoded with pseudo-noise (PN) binary phase codes or modulated with linear frequency (chirp) ramps (Margot, 2001). In both cases, the achievable range resolution is inversely proportional to the bandwidth of the transmitted waveform.

NASA funded a major upgrade of the Arecibo Planetary Radar in the late 1990s, which included a $1 \mathrm{MW}$ S-band transmitter that can support $25 \mathrm{MHz}$ bandwidth modulation (Goldsmith, 1996). In practice, $7.5 \mathrm{~m}$ resolution images are obtained by encoding the transmitted waveform with a $0.05-\mu \mathrm{s}-$ baud $\mathrm{PN}$ code, corresponding to a bandwidth of $20 \mathrm{MHz}$. However, the data acquisition systems available after the upgrade were limited to sampling rates of $5 \mathrm{MHz}$, a factor of four lower than required for maximum resolution. This

\footnotetext{
${ }^{1}$ Corresponding author.
} 
situation prompted the development of a baseband recording system for use at Arecibo, GBT, and other receiving sites. This data-taking system was subsequently deployed at Goldstone as well.

\section{Design Considerations}

Radar echoes in two orthogonal polarizations are detected by low-noise receivers. The receiver signals are amplified, filtered, and typically converted to baseband prior to digitization for computer storage and processing. Important design considerations include the resolution of the analog-to-digital (A/D) converter and the number of bits that are retained for subsequent processing. The data rate is given by

$$
d=2 \times n_{\text {pol }} \times n_{\text {bits }} \times f_{s},
$$

where $n_{\text {pol }}$ is the number of recorded polarizations, $n_{\text {bits }}$ is the number of recorded bits, $f_{s}$ is the sampling frequency in $\mathrm{Hz}$, and the factor of 2 indicates that both the in-phase and quadrature components of the voltage signal are sampled, i.e., a complex quantity. For 8-bit sampling of 2 polarizations at $20 \mathrm{MHz}$, the data rate is $640 \mathrm{Mb} / \mathrm{s}$ or $80 \mathrm{MB} / \mathrm{s}$, which was prohibitive after the Arecibo upgrade.

Fortunately, the first processing step in planetary radar imaging is a range compression operation implemented as a digital correlation. Hagen \& Farley (1973) demonstrated that digital correlations can be carried out effectively with two-, three-, and four-level sampling of the input signals, resulting in surprisingly low degradation of the signal-to-noise ratio (SNR) compared to that obtained with a finely quantized signal. For instance, the ratio of the power obtained with a two-bit (four-level) sampler to the power obtained with an ideal correlator exceeds $88 \%$ for a wide range of quantizer threshold settings (Kogan, 1998). In particular, setting the sampling thresholds at zero and \pm 0.996 times the standard deviation of the input voltage yields a correlator efficiency of $88.12 \%$ with integer output levels of $\{-3,-1,+1,+3\}$ (Schwab, 1986). The design requirement was therefore established at 2-bit sampling of 2 polarizations at $20 \mathrm{MHz}$, or a data rate of $20 \mathrm{MB} / \mathrm{s}$.

The requirements for continuous sampling at low bit resolution are unlike those of most scientific or industrial applications. Commercial data acquisition products typically prize sampling resolutions of at least 8 bits and provide no straightforward mechanism for retaining only the most significant bits. This realization led to a custom-built design and implementation for what became known as the Portable Fast Sampler (PFS), with available sampling modes listed in Table 1.

\begin{tabular}{|r|r|r|r|r|}
\hline mode & channels & bits & sampling rate & data rate \\
\hline 0 & 2 & 1 & $(\mathrm{~N} / \mathrm{A})$ & \\
1 & 2 & 2 & $5-40 \mathrm{MHz}$ & $2.5-20 \mathrm{MB} / \mathrm{s}$ \\
2 & 2 & 4 & $5-20 \mathrm{MHz}$ & $5-20 \mathrm{MB} / \mathrm{s}$ \\
3 & 2 & 8 & $5-10 \mathrm{MHz}$ & $10-20 \mathrm{MB} / \mathrm{s}$ \\
4 & 4 & 1 & $(\mathrm{~N} / \mathrm{A})$ & \\
5 & 4 & 2 & $5-20 \mathrm{MHz}$ & $5-20 \mathrm{MB} / \mathrm{s}$ \\
6 & 4 & 4 & $5-10 \mathrm{MHz}$ & $10-20 \mathrm{MB} / \mathrm{s}$ \\
7 & 4 & 8 & $(\mathrm{~N} / \mathrm{A})$ & \\
\hline
\end{tabular}

Table 1. Description of sampling modes.

\section{Hardware Implementation}

The sampling requirement is achieved with two Analog Devices AD9059/PCB, which are dual-channel 8-bit A/D boards with maximum conversion rates of 60 million samples per second. These boards require only $\mathrm{a}+5 \mathrm{~V}$ power supply and a TTL-compatible encode clock. Full-scale on the A/D converters is achieved for input signals of $1 \mathrm{~V}$ peak-to-peak driving a $50 \Omega$ termination. Therefore, input voltages with standard deviations near $0.25 \mathrm{~V}(1 \mathrm{dBm})$ yield optimum two-bit sampling. Digital outputs are TTL compatible. 
In order to emulate 2-, 4- or 8-bit sampling, the $32 \mathrm{~A} / \mathrm{D}$ digital outputs are connected to a programmable logic device (PLD) for bit selection and packing. A device from the Altera MAX 7000S family (EPM7128SLC84-10) with plastic J-lead chip carrier (PLCC) 84-pin packaging is used for this purpose. It requires a $+5 \mathrm{~V}$ power supply and accepts TTL-level input voltages. The supply voltages for internal logic and input buffers (VCCINT) and for output drivers (VCCIO) are both set to $5 \mathrm{~V}$. The selected bits are packed into sixteen data bits that are sent to four differential line drivers (SN75ALS194) for RS-422 transmission over a 7-foot-long twisted pair cable (EDT CAB-AA 016-00427-00).

A parallel interface card (EDT PCI CD-20) is used to transfer the data to computer memory and storage disks at rates of up to $20 \mathrm{MB} / \mathrm{s}$. The interface card is connected to the PCI bus of a rack-mounted computer that runs the Linux operating system. The interface card can handle four general-purpose control outputs (FUNCT0-3), which are used to specify the sampling mode (written to FUNCT1-3) and to toggle a data acquisition enable signal (written to FUNCT0).

Two station clock signals phase-locked to a high-accuracy frequency standard are required. The first signal is a one pulse-per-second (PPS) TTL signal, which is used to trigger the start of the data acquisition. The second signal is a $+13 \mathrm{dBm}(\sim 3 \mathrm{Vpp}$ into $50 \mathrm{Ohms})$ sampling clock of arbitrary frequency, although most observations are conducted with a 5, 10, or $20 \mathrm{MHz}$ sampling clock. The sampling clock is a simple sine wave that is converted to a TTL square wave and distributed to the A/D boards and PLD.

The enclosure for the A/D boards, PLD, and associated electronics is a Lansing Instrument Corp. B2F18-001C, which is 2 rack units (3.47 in), full rack width (16.73 in), and 18 in deep. BNC connectors are used to connect the 1 PPS, sampling clock, and four analog input signals.

\section{Software Implementation}

A computer program (pfs_radar) written in C can be executed from the command line or graphical user interface to control the operation of the data-taking system. Command-line options enable specification of the sampling mode $(-\mathrm{m})$, recording duration in seconds (-secs), and start time (-start yyyy, $\mathrm{mm}, \mathrm{dd}, \mathrm{hh}, \mathrm{mm}, \mathrm{ss})$ specified in Universal Time. The computer clock is synchronized to accurate time servers with the Network Time Protocol (NTP).

Planetary radar observations require extreme $(<10 \mathrm{~ns})$ timing precision. Upon execution of the datataking program, the computer allocates ring buffer memory, opens log and data files, then suspends execution until $0.5 \mathrm{~s}$ before the desired start time. At that point, the data acquisition enable bit (FUNCT0) is activated, which signals to the PLD that data acquisition will commence on the upcoming rising edge of the 1 PPS signal.

When the rising edge of the 1 PPS is detected while the data acquisition bit is enabled, the PLD toggles the Input Data Valid (IDV) bit, which instructs the 16-bit interface card to transfer data to PCI bus memory. The PLD is programmed to generate a Receive Timing (RXT) signal for use by the interface card, which stores inputs only at the rising edge of the RXT signal. Data are transferred 16 bits at a time until the $\mathrm{C}$ program detects that the end time of data-acquisition has been reached. At that point, the data acquisition bit is deactivated, ring buffers are cleared, the log file is flushed, and the whole process can start over.

Data files contain exclusively the raw data in packed format. Each data file name encodes the receiver start time in the format datayyyymmddhhmmss. Ancillary information about sampling mode, buffer sizes, data rates, etc. are stored in the separate ASCII log file. Data-taking progress can be monitored remotely by examining this log file.

Most radar observations proceed with multiple transmit/receive cycles, where the timing of the receive cycles can be calculated in advance. Many observations use a simple script that repeatedly invokes the data-taking program with the value of the next receive start time.

Ancillary programs enable data inspection and analysis in near real time. One can display histograms and statistics of the input data with pfs_hist and pfs_stats. Unpacking and downsampling of the data can be done with the programs pfs_unpack and pfs_downsample. Spectral analysis is facilitated with pfs_fft. The data-taking software and all ancillary programs are available on GitHub at https://github.com/UCLARADAR-Group/pfs. 


\section{Applications}

The data-acquisition system was installed at Arecibo (2 units), the Green Bank Telescope (2 units), and Goldstone (4 units). It has been used to acquire most of the radar echoes at Arecibo (2000-present), Goldstone (2001-2014), and Green Bank (2001-2017).

The system was used for the first radar detection of a Solar System object at NASA's Deep Space tracking station (DSS-63) in Madrid, Spain. The asteroid 6489 Golevka was detected on 4 June 1999 with the expected Doppler bandwidth and at the expected frequency. Observations of the same asteroid were also obtained at Arecibo in 2003 and led to the first detection of the Yarkovsky orbital drift (Chesley et al., 2003).

Arecibo radar images of asteroid 1999 JM8 were obtained at $15 \mathrm{~m}$ resolution with the data-taking system on 1-9 August 1999. At the time, these images were the highest resolution images of an asteroid ever obtained (Figure 1). This distinction was lost when the NEAR-Shoemaker spacecraft lowered its orbit sufficiently close to asteroid 433 Eros. Analysis of the radar data revealed that the asteroid has an effective diameter of $7 \mathrm{~km}$ and a non-principal-axis rotation with a dominant periodicity near 7 days (Benner et al., 2002).

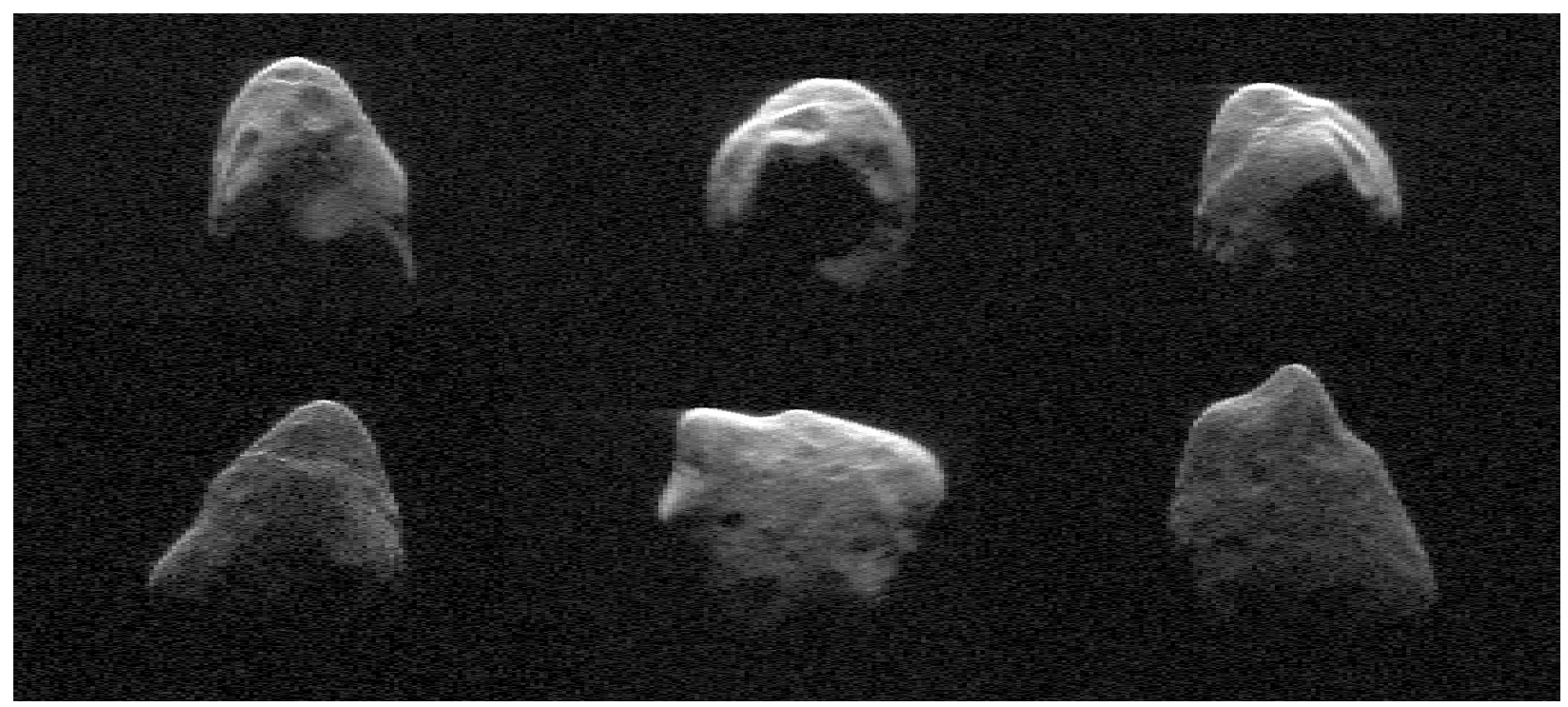

Fig. 1. This sequence of $15 \mathrm{~m}$ resolution Arecibo radar images show asteroid 1999 JM8 on consecutive days.

Observations of the Moon with Arecibo transmitting at $2380 \mathrm{MHz}$ and the $25 \mathrm{~m}$ VLBA antenna in St-Croix receiving were conducted on 19-21 November 2000. The data-taking system was used to record images at $30 \mathrm{~m}$ resolution (Figure 2).

The data-taking system enabled some of the first scientific observations at the Green Bank Telescope. Arecibo-GBT radar images of asteroid 2001 EC16 and Venus at $15 \mathrm{~m}$ and 150-300 m resolutions, respectively, were obtained on 24-26 March 2001. Because the round-trip light-time to the asteroid was 11 s, and because it takes several seconds to switch between transmit and receive modes, monostatic observations would have limited the frequency resolution to the reciprocal of the $\sim 5 \mathrm{~s}$ receive time, approximately $0.2 \mathrm{~Hz}$. Continuous transmission from Arecibo and continuous reception at the GBT for several minutes enabled the acquisition of spectra with two orders of magnitude better frequency resolution. One goal of the Venus observations was to obtain topographic maps with a 3D interferometric technique (Margot et al., 2000).

Other notable radar observations from Arecibo include Saturn's rings in 2001, 2001, and 2003 (Nicholson et al., 2005), binary asteroid $1999 \mathrm{KW} 4$ at $7.5 \mathrm{~m}$ resolution in 2001 (Ostro et al., 2006), and many observations of the Moon (e.g., Campbell et al., 2007).

The sampling system has been used in a Goldstone-GBT configuration to produce high-precision 


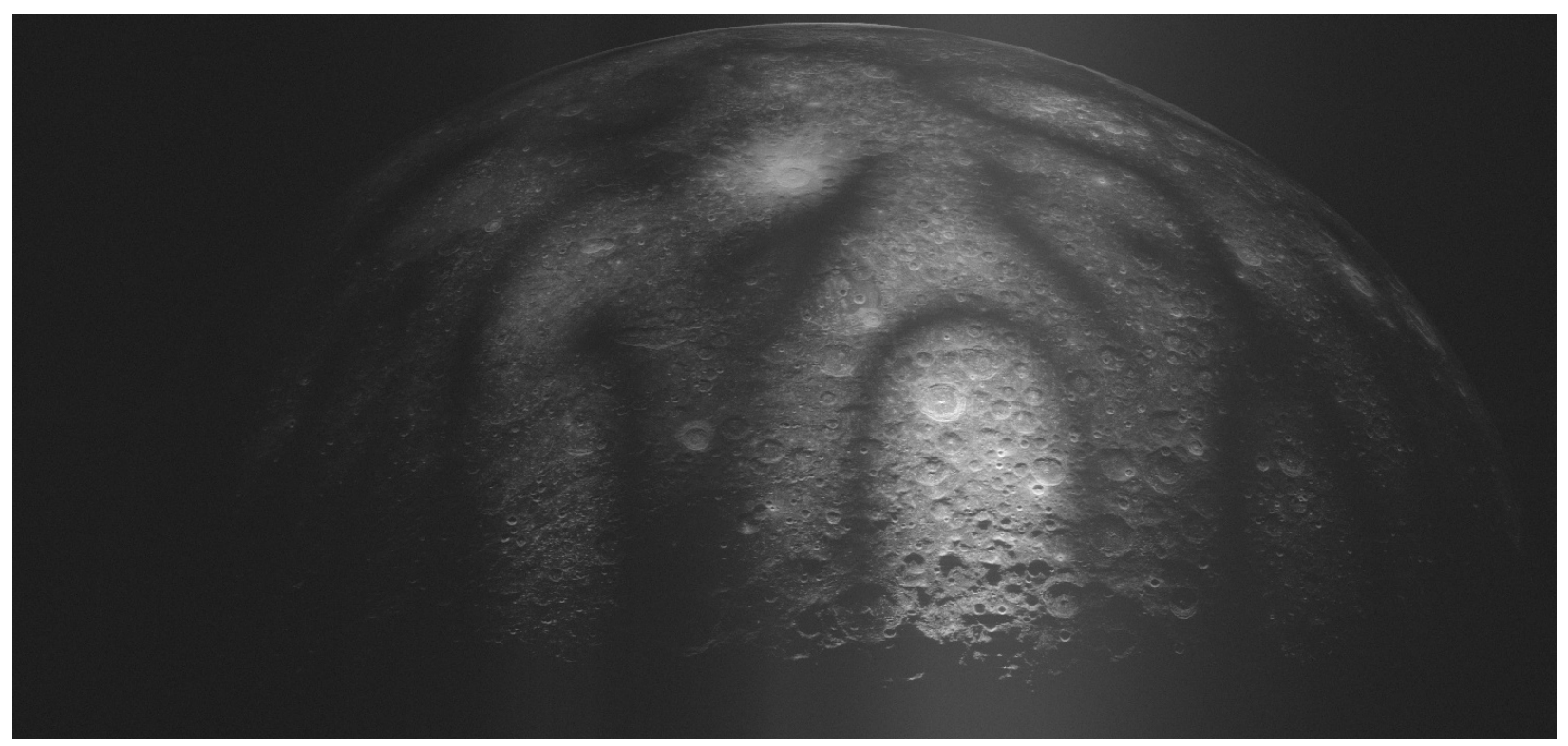

Fig. 2. This Arecibo to St-Croix radar image shows the beam pattern of the Arecibo telescope painted on the surface of the Moon. The main lobe and first null are visible around the south polar region. Echoes from the rest of the Moon appear through the sidelobes of the beam pattern.

measurements of planetary spin states. The observations revealed that Mercury has a liquid outer core (Margot et al., 2007) and enabled a measurement of the size of its core (Margot et al., 2012). They also enabled the first measurement of the spin precession rate of Venus and revealed that Venus exhibits lengthof-day variations of tens of minutes (Margot et al., 2021).

\section{Acknowledgments}

JLM is funded in part by NASA grants 80NSSC18K0850 and 80NSSC19K0870. I thank Jeff Hagen and Joseph Jao for software contributions. The data-taking system was initially funded by the National Astronomy and Ionosphere Center.

\section{References}

Benner, L. A. M., Busch, M. W., Giorgini, J. D., Taylor, P. A. \& Margot, J. L. [2015] "Radar Observations of Near-Earth and Main-Belt Asteroids," Asteroids IV, eds. Michel, P., DeMeo, F. E. \& Bottke, W. F. (Univ. of Arizona Press), p. 165.

Benner, L. A. M., Ostro, S. J., Nolan, M. C., Margot, J. L., Giorgini, J. D., Hudson, R. S., Jurgens, R. F., Slade, M. A., Howell, E. S., Campbell, D. B. \& Yeomans, D. K. [2002] Meteoritics and Planetary Science 37, 779.

Butrica, A. J. [1996] To See the Unseen, NASA SP: 4218 (NASA).

Campbell, B. A., Campbell, D. B., Margot, J. L., Ghent, R. R., Nolan, M., Chandler, J., Carter, L. M. \& Stacy, N. J. S. [2007] IEEE Transactions on Geoscience and Remote Sensing 45, 4032, doi:10.1109/TGRS.2007.906582.

Campbell, D. B., Campbell, B. A., Carter, L. M., Margot, J.-L. \& Stacy, N. J. S. [2006] Nature 443, 835, doi:10.1038/nature05167.

Campbell, D. B., Hudson, R. S. \& Margot, J. L. [2002] "Advances in planetary radar astronomy," Review of Radio Science, ed. W. Ross Stone (URSI).

Chesley, S. R., Ostro, S. J., Vokrouhlický, D., Čapek, D., Giorgini, J. D., Nolan, M. C., Margot, J. L., Hine, A. A., Benner, L. A. M. \& Chamberlin, A. B. [2003] Science 302, 1739, doi:10.1126/science.1091452.

Evans, J. V. \& Hagfors, T. (eds.) [1968] Radar Astronomy (McGraw-Hill, New York).

Giorgini, J. D., Ostro, S. J., Benner, L. A. M., Chodas, P. W., Chesley, S. R., Hudson, R. S., Nolan, M. C., Klemola, A. R., Standish, E. M., Jurgens, R. F., Rose, R., Chamberlin, A. B., Yeomans, D. K. \& Margot, J. L. [2002] Science 296, 132, doi:10.1126/science.1068191.

Goldsmith, P. F. [1996] IEEE Potentials 15, 38.

Hagen, J. B. \& Farley, D. T. [1973] Radio Science 8, 775, doi:10.1029/RS008i008p00775. 
Kogan, L. [1998] Radio Science 33, 1289, doi:10.1029/98RS02202.

Margot, J. L. [2001] "Planetary Radar Astronomy with Linear FM (chirp) Waveforms," Arecibo Technical and Operations Memo Series 2001-09, Arecibo Observatory, URL https://www.naic.edu/ astro/aotms/misc/chirp. pdf.

Margot, J. L., Campbell, D. B., Giorgini, J. D., Jao, J. S., Snedeker, L. G., Ghigo, F. D. \& Bonsall, A. [2021] submitted .

Margot, J. L., Campbell, D. B., Jurgens, R. F. \& Slade, M. A. [1999] J. Geophys. Res. 104, 11875, doi:10.1029/1998JE900047.

Margot, J. L., Campbell, D. B., Jurgens, R. F. \& Slade, M. A. [2000] IEEE Trans. Geoscience and Remote Sensing 38, 1122, doi:10.1109/36.841991.

Margot, J. L., Nolan, M. C., Benner, L. A. M., Ostro, S. J., Jurgens, R. F., Giorgini, J. D., Slade, M. A. \& Campbell, D. B. [2002] Science 296, 1445, doi:10.1126/science.1072094.

Margot, J. L., Peale, S. J., Jurgens, R. F., Slade, M. A. \& Holin, I. V. [2007] Science 316, 710, doi:10.1126/science.1140514.

Margot, J. L., Peale, S. J., Solomon, S. C., Hauck, S. A., II, Ghigo, F. D., Jurgens, R. F., Yseboodt, M., Giorgini, J. D., Padovan, S. \& Campbell, D. B. [2012] Journal of Geophysical Research (Planets) 117, E00L09, doi:10.1029/2012JE004161.

Naidu, S. P., Benner, L. A. M., Margot, J. L., Busch, M. W. \& Taylor, P. A. [2016] Astron. J. 152, 99, doi:10.3847/0004-6256/152/4/99.

Nicholson, P. D., French, R. G., Campbell, D. B., Margot, J.-L., Nolan, M. C., Black, G. J. \& Salo, H. J. [2005] Icarus 177, 32, doi:10.1016/j.icarus.2005.03.023.

Ostro, S. J. [1993] Rev. Modern Phys. 65, 1235, doi:10.1103/RevModPhys.65.1235.

Ostro, S. J., Hudson, R. S., Benner, L. A. M., Giorgini, J. D., Magri, C., Margot, J. L. \& Nolan, M. C. [2002] "Asteroid radar astronomy," Asteroids III, eds. Bottke, W. F., Cellino, A., Paolicchi, P. \& Binzel, R. P. (Univ. of Arizona Press), p. 151.

Ostro, S. J., Hudson, R. S., Nolan, M. C., Margot, J. L., Scheeres, D. J., Campbell, D. B., Magri, C., Giorgini, J. D. \& Yeomans, D. K. [2000] Science 288, 836, doi:10.1126/science.288.5467.836.

Ostro, S. J., Margot, J. L., Benner, L. A. M., Giorgini, J. D., Scheeres, D. J., Fahnestock, E. G., Broschart, S. B., Bellerose, J., Nolan, M. C., Magri, C., Pravec, P., Scheirich, P., Rose, R., Jurgens, R. F., De Jong, E. M. \& Suzuki, S. [2006] Science 314, 1276, doi:10.1126/science.1133622.

Schwab, F. [1986] "Two-bit correlators: Miscellaneous results," VLBA Correlator Memo 75, National Radio Astronomy Observatory.

Taylor, P. A., Margot, J. L., Vokrouhlický, D., Scheeres, D. J., Pravec, P., Lowry, S. C., Fitzsimmons, A., Nolan, M. C., Ostro, S. J., Benner, L. A. M., Giorgini, J. D. \& Magri, C. [2007] Science 316, 274, doi:10.1126/science.1139038. 